\title{
Modeling Family 2 Cystatins and their interaction with Papain
}

Suman Kumar Nandy ${ }^{1}$, Rajabrata Bhuyan ${ }^{1,2}$, Alpana Seal ${ }^{1,2^{*}}$

${ }^{1}$ Department of Biochemistry and Biophysics, University of Kalyani, Kalyani-741235, India.

${ }^{2}$ Bioinformatics Infrastructure Facility, University of Kalyani, Kalyani-741235, India.

Supplementary Material

\section{Table SI}

Result of Secondary structure analysis

\begin{tabular}{|c|c|c|c|c|c|c|}
\hline & & Alpha helix & $\begin{array}{c}\text { Extended } \\
\text { strand }\end{array}$ & $\begin{array}{c}\text { Random } \\
\text { coil }\end{array}$ & Beta Turn & $\begin{array}{c}\text { Ambiguous } \\
\text { states }\end{array}$ \\
\hline \multirow{7}{*}{ Cystatin M/E } & DSC & $33.88 \%$ & $10.74 \%$ & $55.37 \%$ & & \\
\hline & GOR4 & $32.23 \%$ & $26.45 \%$ & $41.32 \%$ & & \\
\hline & HNN & $28.93 \%$ & $15.70 \%$ & $55.37 \%$ & & \\
\hline & PHD & $35.54 \%$ & $16.53 \%$ & $47.93 \%$ & & \\
\hline & Predator & $34.71 \%$ & $19.83 \%$ & $45.45 \%$ & & \\
\hline & SOPMA & $42.98 \%$ & $12.40 \%$ & $39.67 \%$ & & \\
\hline & Sec.Cons. & $32.23 \%$ & $9.09 \%$ & $48.76 \%$ & & $9.92 \%$ \\
\hline \multirow{7}{*}{ Cystatin S } & DSC & $22.31 \%$ & $17.36 \%$ & $60.33 \%$ & & \\
\hline & GOR4 & $32.23 \%$ & $23.14 \%$ & $44.63 \%$ & & \\
\hline & HNN & $35.54 \%$ & $16.53 \%$ & $47.93 \%$ & & \\
\hline & PHD & $24.79 \%$ & $22.31 \%$ & $52.89 \%$ & & \\
\hline & Predator & $34.71 \%$ & $10.74 \%$ & $54.55 \%$ & & \\
\hline & SOPMA & $32.23 \%$ & $14.05 \%$ & $49.59 \%$ & & \\
\hline & Sec.Cons. & $27.27 \%$ & $14.88 \%$ & $52.89 \%$ & & $4.96 \%$ \\
\hline \multirow{6}{*}{ Cystatin SA } & DSC & $23.97 \%$ & $20.66 \%$ & $55.37 \%$ & & \\
\hline & GOR4 & $36.36 \%$ & $19.01 \%$ & $44.63 \%$ & & \\
\hline & $\mathrm{HNN}$ & $38.84 \%$ & $16.53 \%$ & $44.63 \%$ & & \\
\hline & PHD & $27.27 \%$ & $18.18 \%$ & $54.55 \%$ & & \\
\hline & Predator & $35.54 \%$ & $10.74 \%$ & $53.72 \%$ & & \\
\hline & SOPMA & $32.23 \%$ & $15.70 \%$ & $47.11 \%$ & $4.96 \%$ & \\
\hline
\end{tabular}

* Corresponding Author:

Phone: +913325823405

Fax: +913325828282

E-mail: aseal@klyuniv.ac.in 


\begin{tabular}{|c|c|c|c|c|c|c|}
\hline & Sec.Cons. & $28.93 \%$ & $14.05 \%$ & $49.59 \%$ & & $7.44 \%$ \\
\hline \multirow{7}{*}{ Cystatin SN } & DSC & $20.66 \%$ & $17.36 \%$ & $61.98 \%$ & \multirow{7}{*}{$4.13 \%$} & \\
\hline & GOR4 & $28.93 \%$ & $19.83 \%$ & $51.24 \%$ & & \\
\hline & HNN & $33.88 \%$ & $15.70 \%$ & $50.41 \%$ & & \\
\hline & PHD & $27.27 \%$ & $19.01 \%$ & $53.72 \%$ & & \\
\hline & Predator & $33.06 \%$ & $10.74 \%$ & $56.20 \%$ & & \\
\hline & SOPMA & $29.75 \%$ & $16.53 \%$ & $49.59 \%$ & & \\
\hline & Sec.Cons. & $29.75 \%$ & $13.22 \%$ & $51.24 \%$ & & $5.79 \%$ \\
\hline \multirow{7}{*}{ Cystatin C } & DSC & $30.14 \%$ & $10.27 \%$ & $59.59 \%$ & & \\
\hline & GOR4 & $34.25 \%$ & $15.07 \%$ & $50.68 \%$ & & \\
\hline & HNN & $38.36 \%$ & $10.96 \%$ & $50.68 \%$ & & \\
\hline & PHD & $39.73 \%$ & $16.44 \%$ & $43.84 \%$ & & \\
\hline & Predator & $30.82 \%$ & $4.11 \%$ & $65.07 \%$ & \multirow{3}{*}{$6.16 \%$} & \\
\hline & SOPMA & $33.56 \%$ & $15.75 \%$ & $44.52 \%$ & & \\
\hline & Sec.Cons. & $31.51 \%$ & $10.96 \%$ & $52.74 \%$ & & $4.79 \%$ \\
\hline
\end{tabular}



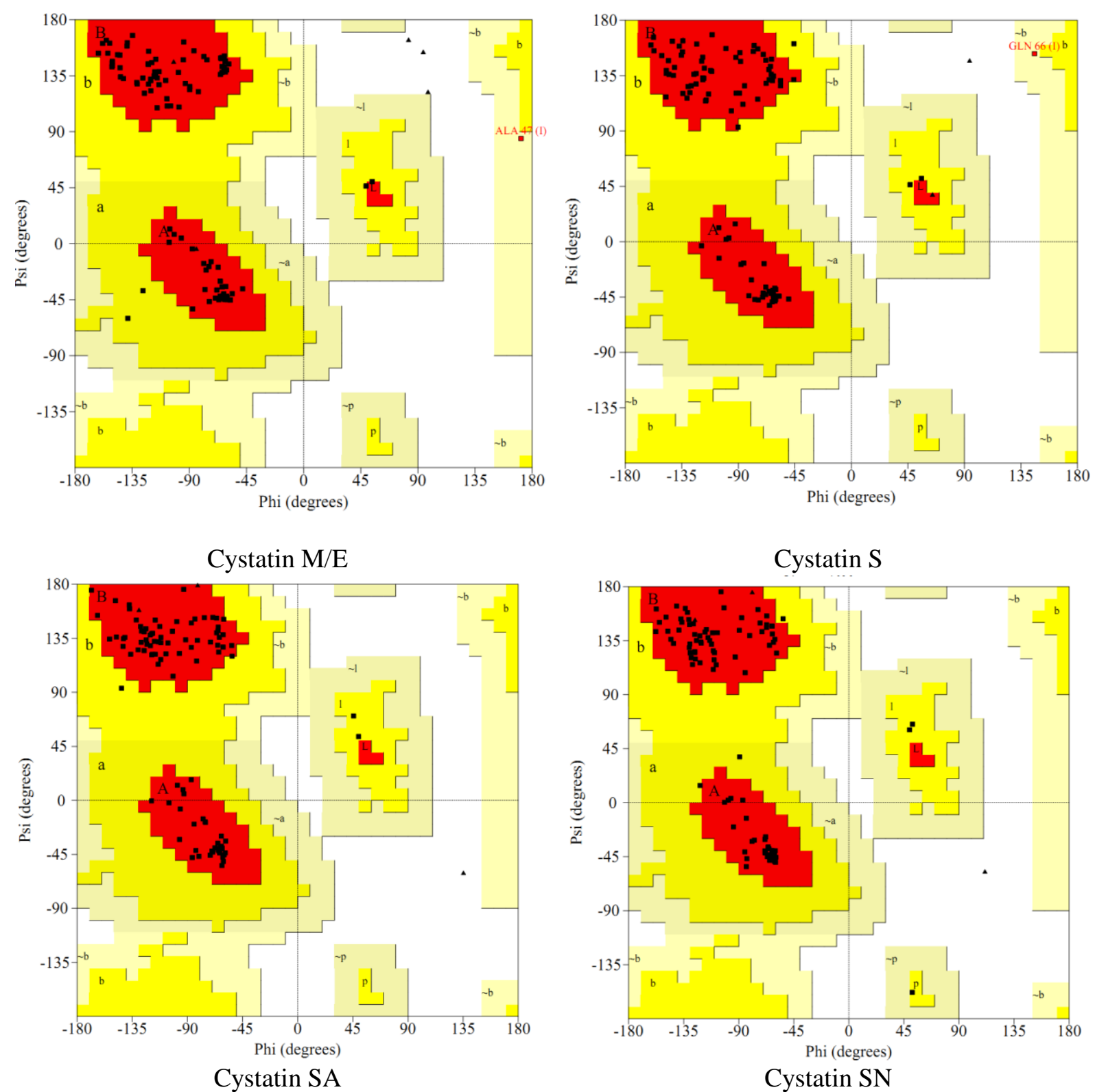

Figure S1: Ramachandran Plot of all the final four models by PROCHECK program. 


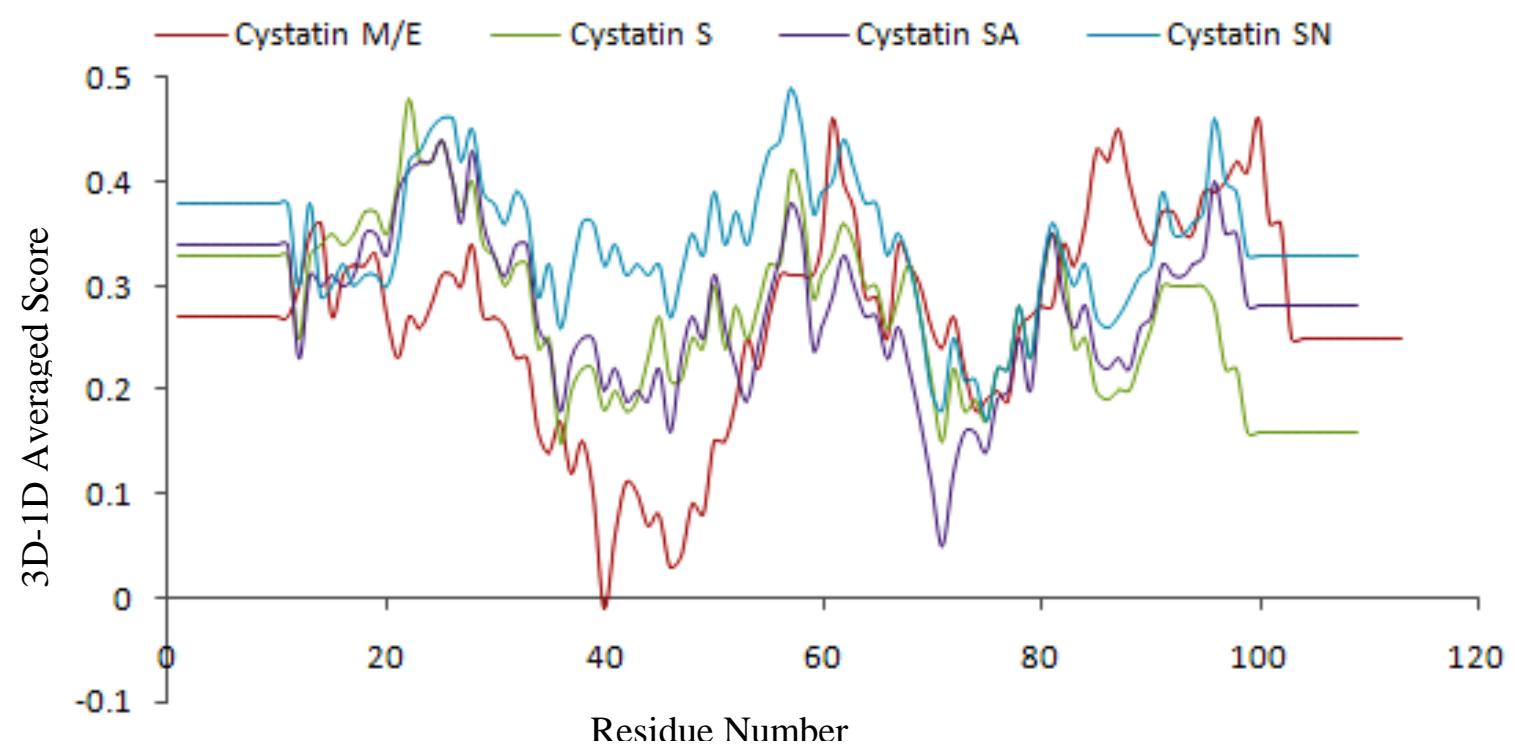

Figure S2: Verify3D validation report of the refined models.

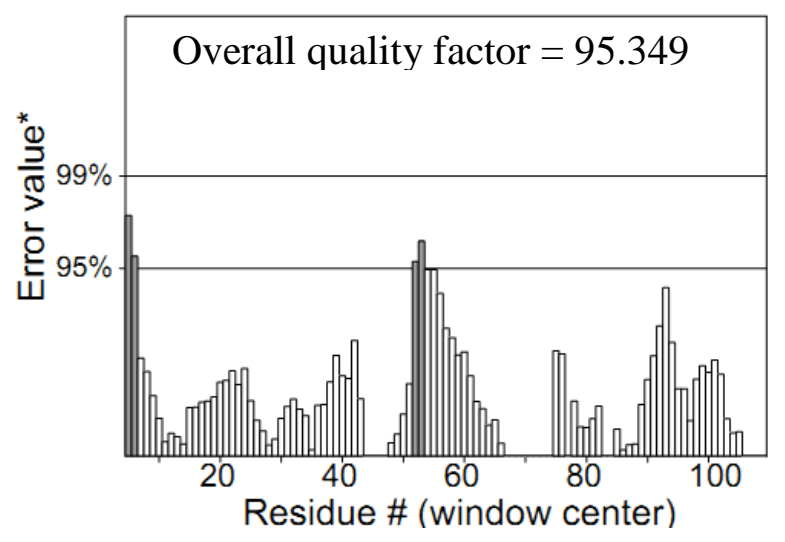

Cystatin M/E

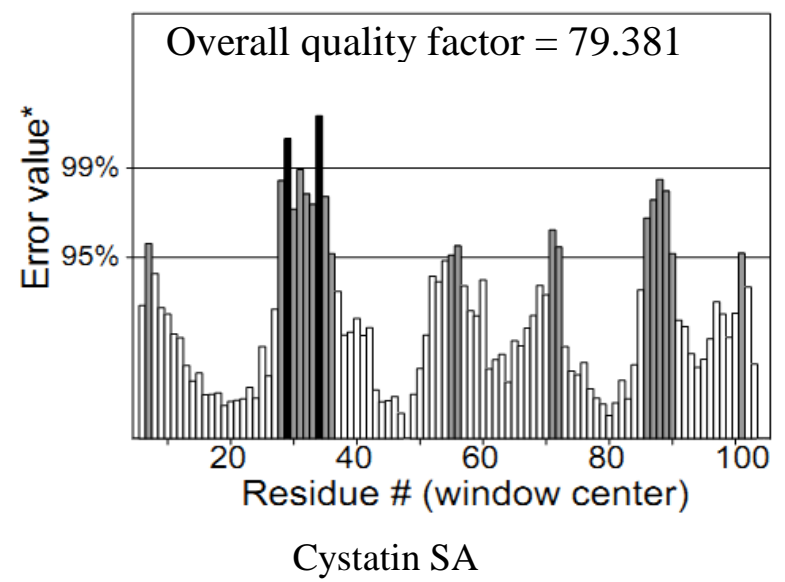

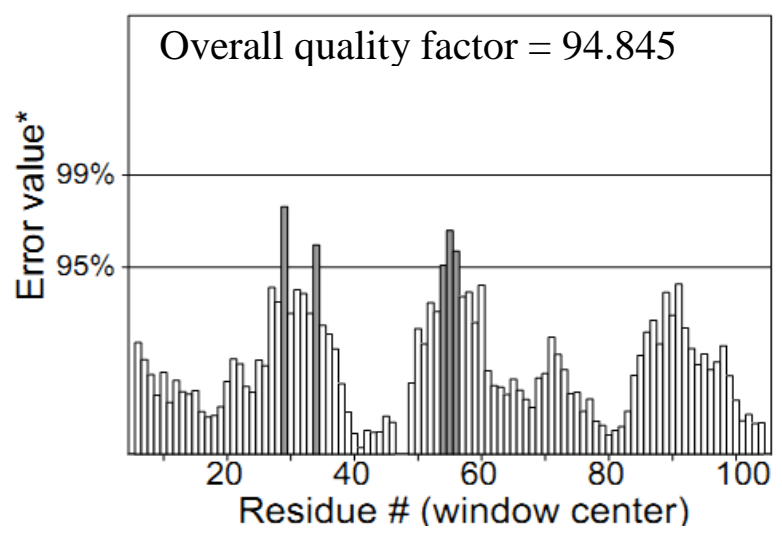

Cystatin S

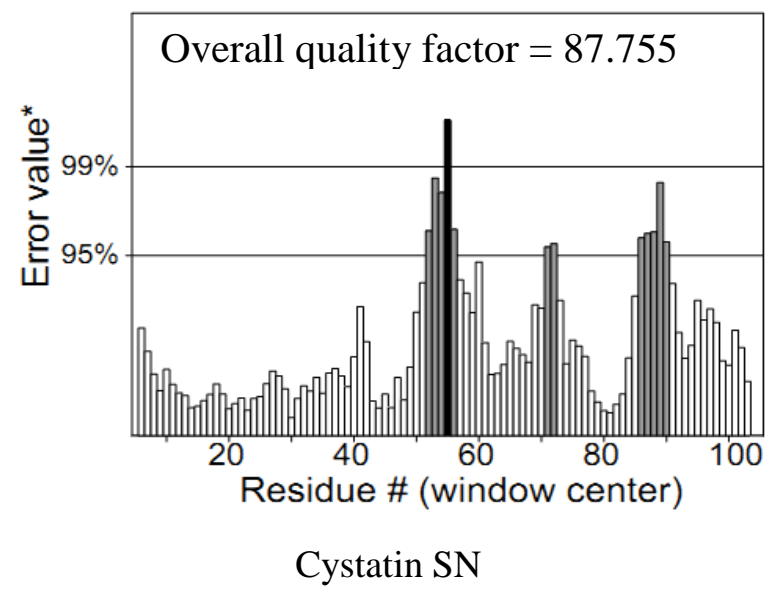

Figure S3: 3D profile scores of final structures using ERRAT Server. 


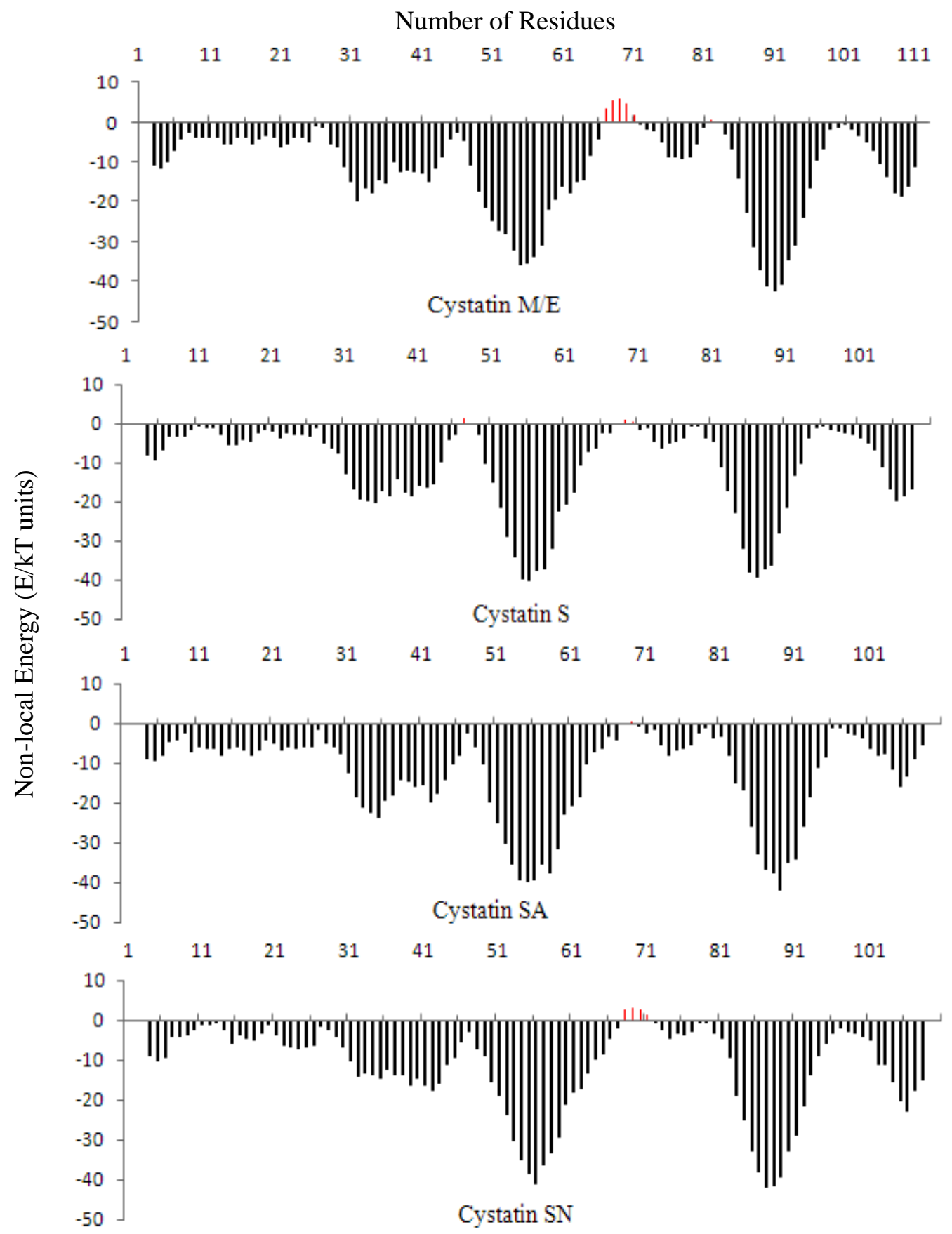

Figure S4: ANOLEA plot, negative values (black) designate residues in a favorable environment and positive values (red) indicate residues in an unfavorable environment. 


\section{Table SII}

Interaction Energy of (IE $>5 \mathrm{kcal} / \mathrm{mol}$ ) interface residues of Papain-Cystatin Complexes

\begin{tabular}{|c|c|c|c|c|c|c|c|c|c|c|c|c|}
\hline \multirow{2}{*}{ Residue } & \multicolumn{3}{|c|}{ Papain-Cystatin M/E } & \multicolumn{3}{|c|}{ Papain-Cystatin S } & \multicolumn{3}{|c|}{ Papain-Cystatin SA } & \multicolumn{3}{|c|}{ Papain-Cystatin SN } \\
\hline & IE & VDW & E_IE & IE & VDW & E_IE & IE & VDW & E_IE & IE & VDW & E_IE \\
\hline A:GLN19 & -8.85 & -3.34 & -5.51 & & & & & & & & & \\
\hline A:GLY20 & -9.12 & -2.58 & -6.59 & & & & & & & & & \\
\hline A:SER21 & -7.22 & -5.46 & -1.76 & -7.56 & -2.48 & -5.07 & -14.47 & -1.26 & -13.20 & -17.29 & -2.14 & -15.15 \\
\hline A:CYS22 & & & & $\underline{-6.18}$ & -2.95 & -3.23 & & & & & & \\
\hline A:TYR61 & & & & $\overline{-6.44}$ & -6.03 & -0.41 & -10.05 & -8.07 & -1.98 & $\underline{-12.88}$ & -2.76 & -10.13 \\
\hline A:CYS63 & & & & & & & & & & -5.88 & -1.18 & -4.69 \\
\hline A:ASN64 & $\underline{-15.99}$ & -3.74 & -12.25 & $\underline{-17.36}$ & -7.06 & -10.30 & $\underline{-23.78}$ & -7.46 & -16.32 & -23.19 & -5.87 & -17.32 \\
\hline A:TYR67 & & & & & & & -13.50 & -1.71 & -11.79 & -21.10 & -5.74 & -15.35 \\
\hline A:TRP69 & & & & & & & & & & $\overline{-8.40}$ & -2.14 & -6.26 \\
\hline A:GLN112 & & & & & & & & & & $\overline{-16.19}$ & 2.31 & -18.50 \\
\hline A:GLU135 & $\underline{-43.55}$ & 4.40 & -47.95 & & & & & & & & & \\
\hline A:LYS139 & -38.69 & 1.60 & -40.30 & -47.80 & 5.83 & -53.63 & $\underline{-54.78}$ & 6.42 & -61.20 & & & \\
\hline A:GLN142 & & & & -16.77 & 0.81 & -17.59 & -12.93 & -3.44 & -9.49 & & & \\
\hline A:LYS156 & $\underline{-62.63}$ & 6.63 & -69.26 & $-\underline{-57.25}$ & 0.27 & -57.53 & -66.91 & 0.27 & -67.17 & $\underline{-51.02}$ & -0.81 & -50.22 \\
\hline A:VAL157 & -14.88 & 0.86 & -15.74 & $-\overline{-16.46}$ & -3.67 & -12.79 & & & & -10.93 & -1.47 & -9.46 \\
\hline A:ASP158 & -52.37 & -4.24 & -48.12 & -19.39 & -5.44 & -13.95 & $\underline{-15.64}$ & -6.12 & -9.52 & -10.42 & -4.61 & -5.81 \\
\hline A:HIS159 & -10.36 & -3.96 & -6.40 & $\overline{-5.07}$ & -4.20 & -0.87 & -8.35 & -5.86 & -2.48 & -9.90 & -5.57 & -4.32 \\
\hline A:TRP177 & $\overline{-8.84}$ & -8.09 & -0.75 & & & & -5.49 & -4.35 & -1.14 & & & \\
\hline A:SER205 & & & & & & & & & & $\underline{-15.40}$ & -0.06 & -15.34 \\
\hline I:GLU1 & $\underline{-90.15}$ & 9.05 & -99.20 & & & & & & & & & \\
\hline I:GLY1 & & & & & & & & & & $\underline{-32.45}$ & 2.06 & -34.50 \\
\hline I:ARG3 & $\underline{-69.48}$ & 1.82 & -71.30 & & & & & & & & & \\
\hline I:ASP6 & $\overline{-38.80}$ & 2.71 & -41.51 & & & & -38.80 & 2.71 & -41.51 & & & \\
\hline I:ASP9 & $\underline{-43.95}$ & 3.72 & -47.67 & & & & & & & -44.67 & 4.85 & -49.52 \\
\hline I:GLU43 & & & & $\underline{-40.53}$ & 2.82 & -43.35 & $\underline{-37.88}$ & 1.61 & -39.50 & & & \\
\hline I:GLN44 & & & & & & & $\overline{-12.19}$ & -0.04 & -12.15 & -9.31 & -3.38 & -5.93 \\
\hline I:LEU45 & -8.24 & -6.96 & -1.28 & & & & & & & & & \\
\hline I:THR45 & & & & $\underline{-15.60}$ & 0.95 & -16.55 & & & & $\underline{-16.72}$ & -4.11 & -12.61 \\
\hline I:ILE45 & & & & & & & -5.87 & -4.21 & -1.66 & & & \\
\hline I:VAL46 & $\underline{-14.64}$ & -6.31 & -8.34 & & & & -9.66 & -8.31 & -1.35 & -11.01 & -8.54 & -2.47 \\
\hline I:PHE46 & & & & -12.30 & -10.30 & -2.00 & & & & & & \\
\hline I:ALA47 & $\underline{-11.08}$ & -5.28 & -5.80 & & & & & & & & & \\
\hline I:GLY47 & & & & -9.73 & -1.24 & -8.48 & $\underline{-10.75}$ & -4.84 & -5.91 & $\underline{-11.32}$ & -4.72 & -6.59 \\
\hline I:GLY48 & & & & -7.52 & -2.67 & -4.86 & & & & & & \\
\hline I:LYS50 & $\underline{-17.72}$ & -0.48 & -17.24 & & & & & & & & & \\
\hline I:ASN50 & & & & & & & $\underline{-6.31}$ & -2.85 & -3.45 & -5.66 & -3.20 & -2.46 \\
\hline I:GLU89 & & & & & & & & & & $-\underline{12.63}$ & -0.55 & -12.09 \\
\hline I:TYR91 & & & & $\underline{-15.64}$ & -6.13 & -9.51 & -10.43 & -8.59 & -1.83 & $-\overline{10.18}$ & -8.50 & -1.68 \\
\hline I:PRO94 & & & & -13.30 & -4.13 & -9.17 & -10.79 & -4.84 & -5.95 & -8.09 & -5.33 & -2.76 \\
\hline I:TRP95 & & & & & & & $\underline{-22.83}$ & -15.07 & -7.76 & -8.74 & -10.63 & 1.89 \\
\hline I:GLU96 & & & & & & & -8.66 & -2.30 & -6.36 & & & \\
\hline I:ASP97 & & & & & & & $\underline{-53.47}$ & 5.91 & -59.38 & & & \\
\hline I:ARG98 & & & & & & & -8.44 & -0.87 & -7.57 & $\underline{-32.46}$ & -1.15 & -31.31 \\
\hline I:TRP99 & $\underline{-21.30}$ & -13.13 & -8.17 & & & & & & & & & \\
\hline I:GLN100 & $\overline{-6.83}$ & -3.40 & -3.43 & & & & & & & & & \\
\hline I:SER100 & & & & & & & $\underline{-10.58}$ & -0.08 & -10.50 & & & \\
\hline I:LYS103 & & & & & & & & & & $\underline{-13.70}$ & 0.12 & -13.82 \\
\hline
\end{tabular}

Note: Residues involved in Hydrogen bond (HB) are underlined, and in solvent accessible surface area (SASA) are italicized in IE column. Residues common in all four or three of the four complexes are in bold. Chain A represents Papain and I stand for cystatin. 


\section{Table SIII}

Relative solvent accessible surface areas (\%) of interface residues of Papain-Cystatin Complexes $[\Delta \mathrm{SAS}>30]$

\begin{tabular}{|c|c|c|c|c|}
\hline \multirow{2}{*}{ Residue } & Cystatin M/E & Cystatin S & Cystatin SA & Cystatin SN \\
\hline & $\triangle \mathrm{SASA}(\%)$ & $\triangle$ SASA (\%) & $\triangle$ SASA $(\%)$ & ASASA (\%) \\
\hline A:GLY20 & $\underline{42.1}$ & & & \\
\hline A:SER21 & 76.1 & & 42.9 & $\underline{47.2}$ \\
\hline A:GLY23 & 40.3 & $\underline{37.1}$ & 36.2 & 40 \\
\hline A:TYR61 & & & & $\underline{30.1}$ \\
\hline A:ASN64 & 48.1 & $\underline{55.5}$ & $\underline{62}$ & $\underline{50.5}$ \\
\hline A:ALA137 & 39.7 & & & \\
\hline A:LYS156 & $\underline{37.5}$ & & $\underline{37.1}$ & $\underline{51.7}$ \\
\hline A:VAL157 & & $\underline{31.38}$ & 30.3 & $\underline{30.6}$ \\
\hline A:ASP158 & $\underline{43.9}$ & $\underline{50.51}$ & $\underline{52.9}$ & $\underline{49.2}$ \\
\hline I:GLU1 & 46.2 & & & \\
\hline I:ARG3 & $\underline{39.9}$ & & & \\
\hline I:LEU45 & 59.7 & & & \\
\hline I:THR45 & & $\underline{54.5}$ & & $\underline{57.6}$ \\
\hline I:VAL46 & $\underline{45.1}$ & & 59.6 & 58.2 \\
\hline I:PHE46 & & 46.7 & & \\
\hline I:ALA47 & $\underline{71.3}$ & & & \\
\hline I:GLY47 & & $\underline{36.7}$ & $\underline{52.6}$ & $\underline{52.7}$ \\
\hline I:TYR91 & & & & 33.3 \\
\hline I:PRO94 & & $\underline{46.2}$ & 32.7 & \\
\hline I:TRP95 & & $\underline{56.7}$ & $\underline{59}$ & 39.3 \\
\hline I:TRP99 & 44.4 & & & \\
\hline I:VAL102 & & 45.6 & & 31.7 \\
\hline
\end{tabular}

Note: Residues present in both, HB \& SASA analysis are underlined \& italicized. Residues common in all four or three of the four complexes are in bold. Chain A corresponds to Papain and I denotes cystatin. 


\section{Table SIV}

Hydrogen Bonds ( $\mathrm{A})$ among interface residues of Papain-Cystatin Complexes

\begin{tabular}{|c|c|c|c|c|c|c|c|}
\hline \multicolumn{2}{|c|}{ Papain-Cystatin M/E } & \multicolumn{2}{|c|}{ Papain-Cystatin S } & \multicolumn{2}{|c|}{ Papain-Cystatin SA } & \multicolumn{2}{|c|}{ Papain-Cystatin SN } \\
\hline $\begin{array}{l}\text { A:GLN19:NE2 - } \\
\quad I: A L A 47: O\end{array}$ & 2.94 & $\begin{array}{l}\text { A:LYS139:NZ - } \\
\text { I:ASP97:OD1 }\end{array}$ & 2.86 & $\begin{array}{c}\text { A:CYS25:SG - } \\
\text { I:GLY47:O }\end{array}$ & 3.16 & $\begin{array}{l}\text { A:TYR67:OH - } \\
\text { I:GLU89:OE2 }\end{array}$ & 2.70 \\
\hline $\begin{array}{c}\text { A:CYS25:SG - } \\
I: V A L 46: O\end{array}$ & 3.04 & $\frac{\text { A:LYS139:NZ - }}{\underline{\text { I:ASP97:OD2 }}}$ & $\underline{2.59}$ & $\frac{\text { A:TYR67:OH - }}{\text { I:GLN44:OE1 }}$ & $\underline{2.66}$ & $\begin{array}{l}\text { A:TRP69:NE1 - } \\
\text { I:GLN44:OE1 }\end{array}$ & 2.78 \\
\hline $\begin{array}{l}\text { A:LYS139:NZ - } \\
\text { I:ASP9:OD1 }\end{array}$ & 3.14 & $\begin{array}{l}\text { A:GLN142:NE2 } \\
\text { - I:PRO94:O }\end{array}$ & 2.71 & $\frac{\text { A:LYS139:NZ - }}{\underline{\text { I:ASP97:OD1 }}}$ & $\underline{2.64}$ & $\begin{array}{c}\text { A:LYS156:NZ - } \\
\text { I:ASP9:OD1 }\end{array}$ & 2.82 \\
\hline$\frac{\text { A:LYS139:NZ- }}{\underline{\text { I:ASP9:OD2 }}}$ & $\underline{2.62}$ & $\begin{array}{l}\text { A:GLN142:NE2 } \\
\text { - I:ASP97:OD1 }\end{array}$ & 2.78 & $\frac{\text { A:LYS139:NZ - }}{\underline{\text { I:ASP97:OD2 }}}$ & $\underline{2.64}$ & $\frac{A: L Y S 156: N Z-}{\underline{\text { I:ASP9:OD2 }}}$ & $\underline{2.62}$ \\
\hline$\frac{\text { A:LYS156:NZ }}{\text { I:GLU1:OE1 }}$ & $\underline{2.64}$ & $\frac{\text { A:LYS156:NZ - }}{\underline{\text { I:GLU43:OE1 }}}$ & $\underline{2.64}$ & $\begin{array}{l}\text { A:GLN142:NE2 } \\
\text { - I:TRP95:O }\end{array}$ & 2.88 & $\begin{array}{l}\text { A:HIS159: } \\
\text { - I:GLY4 }\end{array}$ & 2.87 \\
\hline$\frac{A: L Y S 156: N Z}{\underline{I: G L U 1: O E 2}}$ & $\underline{2.65}$ & $\begin{array}{c}\text { A:LYS156:NZ - } \\
\text { I:THR45:OG1 }\end{array}$ & 2.72 & $\begin{array}{l}\text { A:GLN142:NE2 } \\
\text { - I:ASP97:OD2 }\end{array}$ & 3.18 & $\begin{array}{c}\text { I:GLY1:N - } \\
\text { A:GLN112:OE1 }\end{array}$ & $\underline{2.66}$ \\
\hline $\begin{array}{l}\text { A:HIS159:ND1 } \\
\text { - I:VAL46:O }\end{array}$ & 2.82 & $\begin{array}{l}\text { I:GLY47:N - } \\
\text { A:VAL157:O }\end{array}$ & 2.74 & $\frac{A: L Y S 156: N Z-}{\underline{\text { I:ASP6:OD1 }}}$ & $\underline{2.65}$ & $\begin{array}{l}\text { I:GLY1:N - } \\
\text { A:SER205:OG }\end{array}$ & 2.77 \\
\hline $\begin{array}{l}\text { I:GLU1:N - } \\
\underline{A: L Y S 156: O}\end{array}$ & $\underline{2.66}$ & $\begin{array}{l}\text { I:GLY48:N - } \\
A: V A L 157: O\end{array}$ & 2.97 & $\frac{\text { A:LYS156:NZ - }}{\text { I:GLU43:OE1 }}$ & $\underline{2.66}$ & $\begin{array}{c}\text { I:THR45:OG1 - } \\
\text { A:VAL157:O }\end{array}$ & 2.72 \\
\hline $\begin{array}{l}\text { I:GLU1:N- } \\
\text { A:VAL157:O }\end{array}$ & $\underline{2.67}$ & $\begin{array}{c}\text { I:TYR91:OH - } \\
A: A S P 158: O\end{array}$ & 2.78 & $\begin{array}{l}\text { I:GLN44:NE2 - } \\
\text { A:TYR67:OH }\end{array}$ & 3.12 & $\begin{array}{l}\text { I:GLY48:N - } \\
A: A S P 158: O\end{array}$ & 3.20 \\
\hline $\begin{array}{l}\text { I:ARG3:NH1 - } \\
\text { A:GLU135:OE2 }\end{array}$ & 2.81 & $\begin{array}{c}\text { I:TRP95:NE1 - } \\
\text { A:CYS22:O }\end{array}$ & 2.96 & $\begin{array}{l}\text { I:GLY47:N - } \\
\text { A:ASP158:O }\end{array}$ & 3.03 & $\begin{array}{c}\mathrm{I}: A R G 98: \mathrm{NH} 1= \\
A: S E R 21: O\end{array}$ & 2.76 \\
\hline $\begin{array}{c}\underline{I: A R G 3: N H 1}- \\
\underline{A: A S P 158: O D 2}\end{array}$ & 2.72 & $\begin{array}{l}\text { I:LEU101:N - } \\
A: A S N 64: O D 1\end{array}$ & 2.90 & $\begin{array}{l}\text { I:GLY48:N - } \\
A: A S P 158: O\end{array}$ & 3.09 & $\begin{array}{c}\text { I:ARG98:NH2 - } \\
\text { A:CYS63:O }\end{array}$ & 3.02 \\
\hline $\begin{array}{c}\text { I:ARG3:NH2 - } \\
\text { A:GLU135:OE2 }\end{array}$ & $\underline{2.59}$ & & & $\begin{array}{c}\text { I:ASN50:ND2 - } \\
A: A S N 64: O\end{array}$ & 3.02 & $\begin{array}{c}\text { I:ARG98:NH2 - } \\
\text { A:ASN64:OD1 }\end{array}$ & 2.76 \\
\hline $\begin{array}{l}\text { I:LYS50:NZ - } \\
\text { A:ASN64:OD1 }\end{array}$ & 2.76 & & & $\begin{array}{c}\text { I:ARG98:NH2 - } \\
\text { A:SER21:O }\end{array}$ & 2.78 & $\begin{array}{l}\text { I:LYS103:NZ - } \\
\text { A:TYR61:OH }\end{array}$ & 2.83 \\
\hline $\begin{array}{c}\text { I:TRP99:NE1 - } \\
\text { A:GLY20:O }\end{array}$ & 2.86 & & & $\begin{array}{l}\text { I:SER100:OG - } \\
A: A S N 64: O D 1\end{array}$ & 2.74 & $\begin{array}{l}\text { I:LYS103:NZ - } \\
\text { A:TYR67:OH }\end{array}$ & 3.14 \\
\hline
\end{tabular}

Note: Residues present in SASA analysis are italicized and residues involved in SSHB $(<2.7 \AA)$ are underlined. Chain A symbolizes Papain whereas I indicate cystatin. 


\section{Table SV}

Triadic Hydrogen Bonds

\begin{tabular}{|c|c|c|c|}
\hline Papain-Cystatin M/E & Papain-Cystatin S & Papain-Cystatin SA & Papain-Cystatin SN \\
\hline I:ASP9:A:LYS139:I:ASP9 & I:ASP97:A:LYS139:I:ASP97 & A:CYS25:I:GLY47:A:ASP158 & I:GLU89:A:TYR67:I:LYS103 \\
\hline I:GLU1:A:LYS156:I:GLU1 & I:PRO94:A:GLN142:I:ASP97 & I:GLN44:A:TYR67:I:GLN44 & I:ASP9:A:LYS156: I:ASP9 \\
\hline A:VAL157:I:GLU1:A:LYS156 & I:GLU43:A:LYS156:I:THR45 & I:ASP97:A:LYS139:I:ASP97 & A:GLN112:I:GLY1:A:SER205 \\
\hline A:GLU135:I:ARG3:A:ASP158 & I:GLY47:A:VAL157:I:GLY48 & I:ASP97:A:GLN142:I:TRP95 & A:SER21:I:ARG98: A:CYS63 \\
\hline A:CYS25:I:VALA6:A:HIS159 & & I:ASP6:A:LYS156: I:GLU43 & A:TYR67:I:LYS103:A:TYR61 \\
\hline I:ARG3: A:GLU135: I:ARG3 & & I:GLY47:A:ASP158:I:GLY48 & A:CYS63:I:ARG98:A:ASN64 \\
\hline & & I:ASN50:A:ASN64:I:SER100 & \\
\hline
\end{tabular}

Note: Residues present in SASA analysis are italicized and residues involved in SSHB are underlined. Chain A \& I signify Papain and Cystatin respectively. 